\title{
Glass transition properties of PMMA thin shells deposited on rodlike calcium carbonate particles
}

\author{
Takashi Sasaki $^{1}$, Rumi Kuroda ${ }^{1}$, Mitsuhiro Teramoto $^{1}$, Shigeru Yonezawa ${ }^{1}$, Hiromasa Tsuji ${ }^{1}$, Kensuke Sakurai ${ }^{1}$ \\ and Satoshi Irie ${ }^{2}$
}

Rodlike capsules consisting of a calcium carbonate core and a thin shell of poly(methyl methacrylate) (PMMA) were synthesized. The shell thickness $d$ ranged from 8 to $93 \mathrm{~nm}$, and the degree of crosslink $X_{\mathrm{c}}$ of the polymer shell ranged from 0 to $7.0 \mathrm{wt} \%$, where $X_{c}$ is defined as the mass content of the crosslink monomer (ethylene glycol dimethacrylate). The glass transition temperature $T_{\mathrm{g}}$ and the length scale for dynamic heterogeneity at the glass transition $\xi\left(T_{\mathrm{g}}\right)$ for the polymer thin shells were evaluated by temperature-modulated differential scanning calorimetry. It was found that $T_{\mathrm{g}}$ is not altered from the bulk value for $d \geqslant c a .20 \mathrm{~nm}$ for both filled and hollow shells. The thinnest non-crosslinked shell $(d=8 \mathrm{~nm})$ exhibited a lowered $T_{\mathrm{g}}$ from the bulk value. For $\xi\left(T_{\mathrm{g}}\right)$, no distinct dependence on $d$ was observed for non-crosslinked filled shells. The correlation between $\xi\left(T_{\mathrm{g}}\right)$ and $T_{\mathrm{g}}$ was also revealed, which is interpreted in terms of the concept of a cooperatively rearranging region.

Polymer Journal (2011) 43, 464-470; doi:10.1038/pj.2011.7; published online 9 February 2011

Keywords: capsules; crosslink; glass transition; PMMA

\section{INTRODUCTION}

The thermal and mechanical properties of nano-sized polymeric materials have attracted much attention in a wide range of technological areas concerning nano-devices, capsules, nanocomposites, coatings and biomedical applications. From a practical point of view and for fundamental research on soft matter, it is becoming important to understand the origin of the anomalous dynamics of nano-sized and nano-confined polymers, which differ considerably from the bulk materials. ${ }^{1}$ Investigations to clarify the mechanism of such anomalous dynamics have been extensively performed, especially for the glass transition temperature, $T_{\mathrm{g}}$, of ultrathin films, ${ }^{2-7}$ and of confined liquids in nanopores, ${ }^{8,9}$ spheres ${ }^{10}$ and phase separated polymers, ${ }^{11}$ but the subject still seems to be a controversial issue. $T_{\mathrm{g}}$ depends on various factors including size, status of the interface, geometry and experimental method (sample preparation and observation). In addition, some systems show very weak or no size dependence of $T_{\mathrm{g}}$ even below $100 \mathrm{~nm}$. $6,7,10-12$

Researchers have been trying to understand the mechanism of glass transition in terms of the cooperatively rearranging region (CRR), ${ }^{13,14}$ of which the size increases with decreasing temperature toward an underlying thermodynamic transition point, leading to a drastic increase in relaxation time. The segmental relaxation time is supposed to be a function of the configurational entropy of the smallest CRR $s_{\mathrm{c}}{ }^{*}$. The random first-order transition theory, ${ }^{15}$ which was developed recently, also supposes the existence of a CRR, where nucleation for a transition in the CRR is treated based on a mean field assumption.
According to this theory, $s_{\mathrm{c}}{ }^{*}$ depends on temperature, and the relaxation time depends on the configurational entropy per segment, size of CRR, distribution of relaxation times, dynamic fragility and heat capacity difference between the glassy and liquid states. It is crucial to determine how these parameters are affected by the various factors that alter $T_{\mathrm{g}}$ in nano-sized polymers to understand their anomalous glass transition dynamics.

The glass transition behaviors of ultrathin polymer shells deposited onto the surface of rodlike $\mathrm{CaCO}_{3}$ particles (whisker) have been studied, and no $T_{\mathrm{g}}$ deviations from the bulk for both polystyrene and poly(methyl methacrylate) (PMMA) shells without crosslinking were found. ${ }^{12}$ The case for their hollow shells is similar, which seems to be quite inconsistent with the behaviors of ultrathin polymer films reported in the literature. ${ }^{2-5}$ On the other hand, for crosslinked polystyrene shells, increased $T_{\mathrm{g}} \mathrm{s}$ relative to the bulk value have been observed depending on the shell thickness, ${ }^{16,17}$ which has been attributed to the heterogeneous nature of crosslink and also to the unstable configurational structure retained by the crosslink. To elucidate more precisely the dynamics and mechanism of the glass transition in the thin shells of rodlike capsules, shells of various other polymers should be investigated.

In this paper, the glass transition behaviors of PMMA thin shells, deposited on the surface of calcium carbonate whisker particles with different thicknesses and degrees of crosslink, were investigated by temperature-modulated differential scanning calorimetry (TMDSC), and their glass transition behaviors are compared with those of the

${ }^{1}$ Department of Materials Science and Engineering, University of Fukui, Fukui, Japan and ${ }^{2}$ Headquarters for Innovative Society-Academia Cooperation, University of Fukui, Fukui, Japan

Correspondence: Professor T Sasaki, Department of Materials Science and Engineering, University of Fukui, 3-9-1 Bunkyo, Fukui 910 8507, Japan.

E-mail: sasaki@matse.u-fukui.ac.jp

Received 17 November 2010; revised and accepted 27 December 2010; published online 9 February 2011 
polystyrene shells investigated in previous works. ${ }^{16,17}$ To this end, the PMMA capsules were prepared by two different methods: by radical polymerization in an aqueous suspension of the whisker particles and by simple adsorption during bulk polymerization under the dispersion of whisker particles. The latter method yields shells thinner than $10 \mathrm{~nm}$.

\section{EXPERIMENTAL PROCEDURE Sample preparation}

Calcium carbonate whiskers were supplied from Maruo Calcium, Akashi, Japan. Methyl methacrylate (MMA) and the crosslink monomer, ethylene glycol dimethacrylate (EGDMA) were distilled under reduced pressure before use. Encapsulation of the calcium carbonate core was performed by the following two methods: (1) radical polymerization in aqueous suspension of the whisker particles, and (2) adsorption of a PMMA layer during bulk polymerization under the dispersion of whisker particles (referred to as the adsorption method hereafter). A detailed description of method (1) has been presented in previous papers. ${ }^{12,16}$ Co-polymerization of MMA/EGDMA was executed in an aqueous suspension of $\mathrm{CaCO}_{3}$ whisker particles stabilized by laurylbenzenesulfonic acid sodium salt. In method (2), the PMMA shell was deposited during bulk polymerization by simple physical adsorption. This method was applicable only to the preparation of non-crosslinked shells because crosslinking prevents the formation of a thin layer due to apparent gelation. The atom transfer radical polymerization of MMA was performed ${ }^{18,19}$ as follows: $\mathrm{CaCO}_{3}$ whisker particles $(400 \mathrm{mg})$ were suspended in MMA $(10.0 \mathrm{~g})$, and ethyl 2-bromoisobutyrate $(6.4 \mathrm{mg})$, dinonyl-2,2'-bipyridine $(138 \mathrm{mg})$ and $\mathrm{Cu}(\mathrm{I}) \mathrm{Cl}(16.0 \mathrm{mg})$ were added to this suspension. Then, it was immediately degassed under high vacuum. The reaction tube was sealed off after several freeze-pump-thaw cycles. The radical polymerization was executed at room temperature for $96 \mathrm{~h}$. Tetrahydrofuran $(200 \mathrm{ml})$ was added to the reacted mixture, and the whisker particles were separated by centrifugation, washed with methanol several times and finally dried under vacuum at $40^{\circ} \mathrm{C}$ for $24 \mathrm{~h}$. On the other hand, the remaining PMMA/tetrahydrofuran solution that contains no whisker particles was subject to precipitation in $n$-hexane three times, and the obtained bulk PMMA was dried under vacuum at $60^{\circ} \mathrm{C}$ for $24 \mathrm{~h}$. The molecular weight of this bulk PMMA was evaluated by size exclusion chromatography to be $M_{\mathrm{n}}=156 \mathrm{~kg} \mathrm{~mol}^{-1}$, and $M_{\mathrm{w}}=214 \mathrm{~kg} \mathrm{~mol}^{-1}$.

Hollow samples were also prepared from the core/shell capsules obtained above (filled capsules) by simply dissolving the inorganic core in hydrochloric acid. ${ }^{12}$ All of the prepared capsules were washed with methanol and were dried under vacuum for $170 \mathrm{~h}$ at room temperature before the differential scanning calorimetry (DSC) measurements were taken. In this study, the degree of crosslink $X_{\mathrm{c}}$ is defined as

$$
X_{\mathrm{c}}=m_{\mathrm{EGDMA}} /\left(m_{\mathrm{MMA}}+m_{\mathrm{EGDMA}}\right),
$$

where $m_{\mathrm{MMA}}$ and $m_{\mathrm{EGDMA}}$ are the masses of MMA and EGDMA fed in the reaction mixture.

Reference bulk samples were prepared from the hollow particles: hollow samples were first dissolved in tetrahydrofuran, followed by drying (casting) at room temperature. The yielded bulk films were further dried in vacuum at $60^{\circ} \mathrm{C}$ for $168 \mathrm{~h}$.

Morphology of the obtained capsules was examined by transmission electron microscopy by use of a Jeol JEM-2100 $(200 \mathrm{kV})$ (Jeol, Akishima, Japan). The average shell thickness and its standard deviation were evaluated from electron micrographs by measuring at hundreds of different locations of different particles.

\section{Calorimetry}

Glass transition behaviors were investigated by using a Perkin-Elmer Pyris Diamond DSC calorimeter (Perkin-Elmer, Waltham, MA, USA). TMDSC measurements were performed: a saw-tooth modulation with amplitude of $0.5 \mathrm{~K}$ and a period of $60 \mathrm{~s}$ was superimposed on a cooling scan at $-0.2 \mathrm{~K} \mathrm{~min}^{-1}$, which started at a temperature well above $T_{\mathrm{g}}$ (typically $\sim 140^{\circ} \mathrm{C}$ ). The Lissajous plots showed a negligible nonlinear effect of thermal response. To evaluate the heat capacity data around $T_{\mathrm{g}}$, step-scan DSC measurements were performed; each step consisted of a heating stage of $2 \mathrm{~K}$ at $5.0 \mathrm{~K} \mathrm{~min}^{-1}$ followed by a temperature holding segment for $1.5 \mathrm{~min}$. The complex heat capacity was evaluated from the heat flow data of TMDSC for each modulation cycle, and the storage and loss heat capacities $\left(C^{\prime}\right.$ and $C^{\prime \prime}$, respectively) were obtained with respect to temperature. The characteristic length at the glass transition $\xi\left(T_{\mathrm{g}}\right)$ was evaluated from the loss heat capacity trace $C^{\prime \prime}(\omega)$ according to the fluctuation-dissipation theory. ${ }^{20}$ Values of the heat capacity reported in the literature were used for the evaluation of $\Delta\left(1 / C_{\mathrm{V}}\right)$, which was needed to obtain the values of $\xi\left(T_{\mathrm{g}}\right){ }^{21}$ The detailed method of evaluation is described in a previous paper. ${ }^{17}$ To evaluate the standard deviation for the obtained data, multiple measurements (3-10 times) for each DSC scan were performed with different specimens. The loss of sample mass after each DSC scan did not exceed $0.2 \%$ of the polymer mass. As for the hollow capsules, reliable data for $\xi\left(T_{\mathrm{g}}\right)$ could not be obtained because the hollow structure collapsed to some extent after the TMDSC scan. Therefore, in this paper, only the $T_{\mathrm{g}}$ values obtained by the step-scan measurements for the hollow samples are presented. In contrast, the filled capsules were rather stable: they did not exhibit apparent morphological change as observed by the transmission electron microscopy after the TMDSC scan, and a few succeeding TMDSC scans gave almost identical results.

\section{Estimation of interfacial tension}

The interfacial tension $\gamma_{\mathrm{SL}}$ for PMMA and polystyrene (for comparison) on the $\mathrm{CaCO}_{3}$ surface was evaluated by the procedure based on the Fowkes-van OssChaundhury-Good method. ${ }^{22-24} \mathrm{CaCO}_{3}$ whisker particles were pressed at $100 \mathrm{~kg} \mathrm{~cm}^{-2}$ for $10 \mathrm{~min}$ with degassing to make a pellet with a specular surface. Water, glycerol and ethylene glycol were used as probe liquids. The contact angle for the droplet on the $\mathrm{CaCO}_{3}$ pellet surface for these three liquids was measured, and the surface tension parameters for $\mathrm{CaCO}_{3}$ were evaluated by use of the literature values for the three liquids. ${ }^{23}$ Using also the literature values for the surface tension parameters for the polymers, ${ }^{25} \gamma_{\mathrm{SL}}$ was evaluated for $\mathrm{PMMA} / \mathrm{CaCO}_{3}$ and polystyrene/ $\mathrm{CaCO}_{3}$ as

$$
\gamma_{\mathrm{SL}}=\left(\sqrt{\gamma_{\mathrm{S}}^{\mathrm{LW}}}-\sqrt{\gamma_{\mathrm{L}}^{\mathrm{LW}}}\right)^{2}+2\left(\sqrt{\gamma_{\mathrm{S}}^{+} \gamma_{\mathrm{S}}^{-}}+\sqrt{\gamma_{\mathrm{L}}^{+} \gamma_{\mathrm{L}}^{-}}-\sqrt{\gamma_{\mathrm{S}}^{+} \gamma_{\mathrm{L}}^{-}}-\sqrt{\gamma_{\mathrm{L}}^{+} \gamma_{\mathrm{S}}^{-}}\right),
$$

where $\gamma^{\mathrm{LW}}$ is the Lifshitz-van der Waals component of the interfacial tension, $\gamma^{+}$and $\gamma^{-}$are the hydrogen bonding components, and the subscripts $\mathrm{S}$ and $\mathrm{L}$ denote solid and liquid, respectively.

\section{RESULTS AND DISCUSSION}

\section{Morphology}

Figure 1 shows transmission electron microscopy images of the capsules prepared by the suspension polymerization. We see that the outer shells are successfully formed for various $X_{\mathrm{c}} \mathrm{s}$. We infer that in the suspension polymerization, a thin monomer layer is first formed on the surface of whisker particles; then, radical polymerization proceeds in the layer as formed, which results in a polymer shell with a smooth surface. In contrast, polystyrene shells prepared by a similar method exhibit a relatively ragged surface compared with the PMMA shells, and under certain preparation conditions, many spheres are attached to the whisker surface. ${ }^{16,17}$ This is probably due to a higher interfacial energy at the layer/whisker for the polystyrene system. In this case, the formation of spherical micelles tends to be favored rather than formation of an unstable layer on the whisker surface. Contact angle measurements showed that an MMA/PMMA solution (7/3 by weight) exhibits higher wettability on a $\mathrm{CaCO}_{3}$ substrate than a styrene/polystyrene solution. This observation supports the above assumption. The detailed mechanism of radical polymerization of different monomers in the presence of suspended whisker particles will be discussed in a separate paper on the basis of polymerization rate. The adsorption method also yielded core/shell capsules as shown in Figure 2, although the surface of the outer layer is thinner but less smooth than that from the suspension polymerization. 

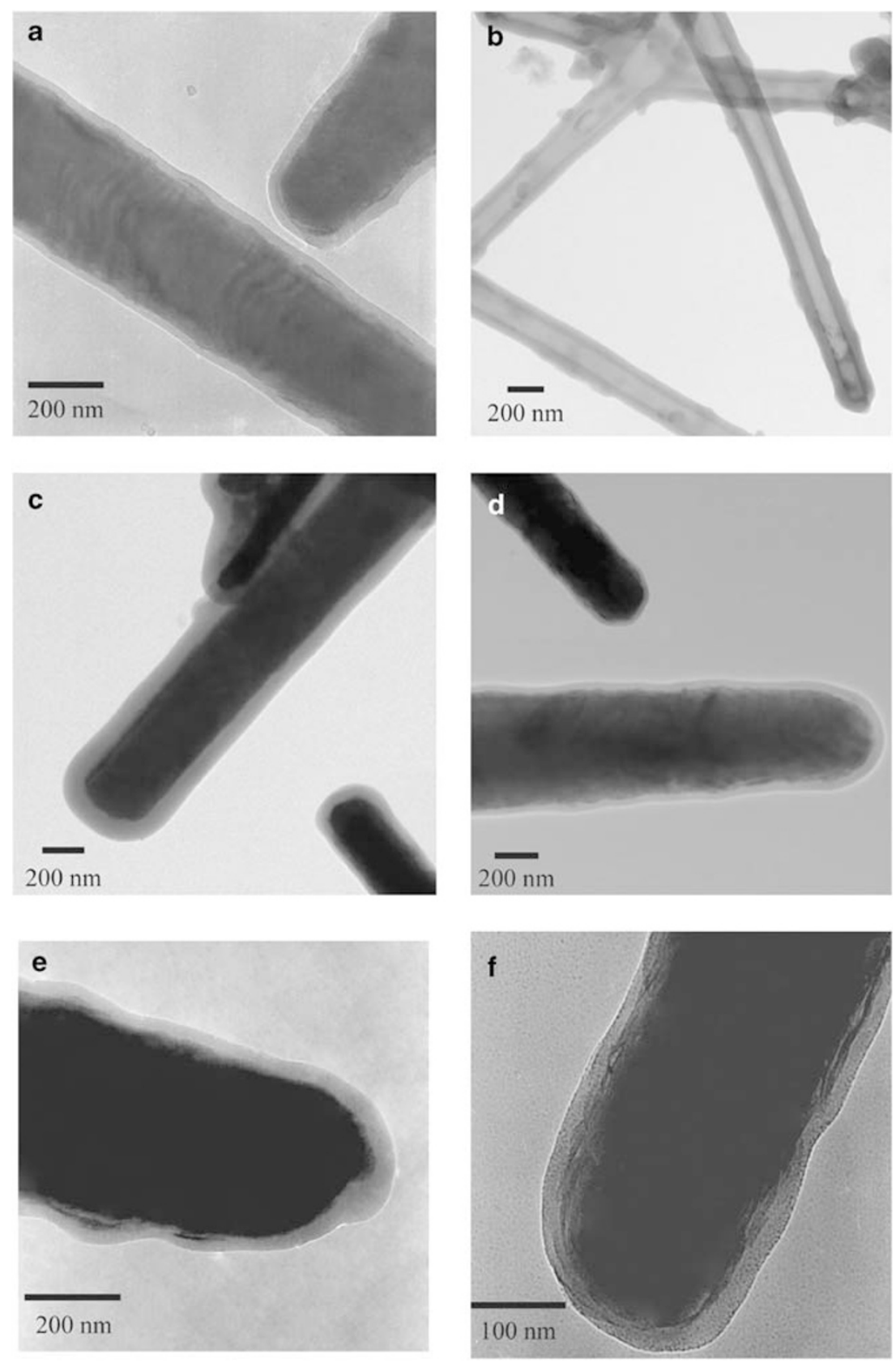

Figure 1 Electron micrographs of (a) a non-crosslinked filled capsule, (b) non-crosslinked hollow capsule, (c) a non-crosslinked filled capsule with a thicker shell $(d=93 \mathrm{~nm})$, (d) a filled capsule with $X_{c}=1.0 \mathrm{wt} \%$, (e) a filled capsule with $X_{c}=3.0 \mathrm{wt} \%$ and (f) a filled capsule with $X_{c}=7.0 \mathrm{wt} \%$.

In the suspension polymerization method, the shell thickness can be controlled by the amount of monomer fed, as was reported for polystyrene capsules. ${ }^{16,17}$ The thickness of the outer PMMA shells used in this study is shown in Table 1.

Hollow capsules were successfully obtained from the samples prepared by the suspension polymerization method as shown in Figure 1b. However, no rodlike hollow capsules were obtained from the sample prepared by the adsorption method. It is likely that fragmentation of the shell occurred in the acid treatment, which suggests less surface coverage by the shell from the adsorption method than from the suspension polymerization method. Table 1 shows that the shell thickness $d$ increases after removing the core. This tendency is generally observed for the capsules prepared by the suspension polymerization. The as-polymerized shell is probably in an unstable configurational state, and structural relaxation thus occurs upon removing the core, which results in thickening of the shell. ${ }^{16}$ It is also noted that the thickening tendency is prominent for thinner shells. As for the thickest shell $(d=93 \mathrm{~nm})$, on the other hand, the thickness does not change within the error range; the effect of the unstable structure may be weakened for thicker shells.

\section{Glass transition behavior}

Figure 3 shows typical traces obtained from TMDSC cooling scans. The profile of $C^{\prime \prime}(T)$, which exhibits a peak in the glass transition range, was fitted with a Gaussian function to evaluate the dynamic length scale $\xi\left(T_{\mathrm{g}}\right)$. Figure $4 \mathrm{a}$ shows the $T_{\mathrm{g}}$ difference between the 

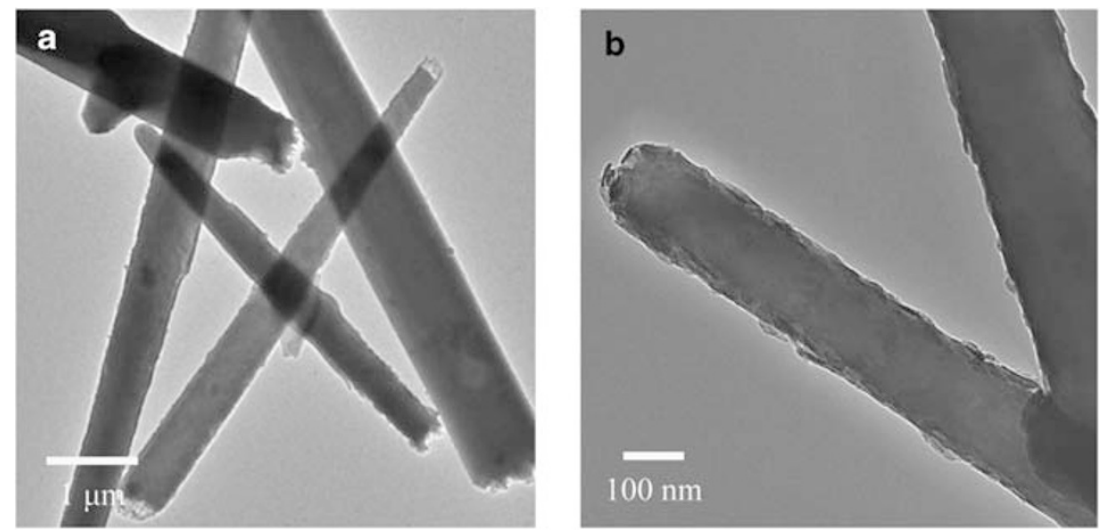

Figure 2 Electron micrographs of a non-crosslinked filled capsule with $d=8 \mathrm{~nm}$ prepared by the adsorption method.

Table 1 Thickness of outer poly(methyl methacrylate) shells

\begin{tabular}{lccc}
\hline & & \multicolumn{2}{c}{$\mathrm{d}(\mathrm{nm})$} \\
\cline { 3 - 4 } Sample & & Filled & Hollow \\
\hline A & $X_{c}(w t \%)$ & $8 \pm 1$ & \\
B & 0 & $23 \pm 5$ & $50 \pm 8$ \\
C & 0 & $36 \pm 4$ & $65 \pm 9$ \\
D & 0 & $93 \pm 19$ & $79 \pm 8$ \\
E & 0 & $22 \pm 4$ & $45 \pm 6$ \\
F & 1.0 & $21 \pm 5$ & $43 \pm 9$ \\
G & 3.0 & $20 \pm 4$ & $42 \pm 6$ \\
H & 5.0 & $17 \pm 3$ & $41 \pm 7$ \\
\hline
\end{tabular}

non-crosslinked filled shell and the corresponding bulk, $\Delta T_{\mathrm{g}}=T_{\mathrm{g}}^{\mathrm{f}}-T_{\mathrm{g}} \mathrm{b}$, plotted against the shell thickness $d$. The result shows no $T_{\mathrm{g}}$ difference from the bulk for $d \geqslant 23 \mathrm{~nm}$, while the shell with $d=8 \mathrm{~nm}$ exhibits lower $T_{\mathrm{g}}$ than the bulk. These results are in contrast to the reported $T_{\mathrm{g}}$ behavior of PMMA ultrathin films: PMMA films with $d=$ ca. $20 \mathrm{~nm}$ on $\mathrm{SiO}_{x}$ substrates have been reported to exhibit $T_{\mathrm{g}}$ shifts of $5-10 \mathrm{~K}$ (ref. 26). No $T_{\mathrm{g}}$ shift was observed for non-crosslinked polystyrene shells, ${ }^{16}$ which is again in contradistinction to the drastic $T_{\mathrm{g}}$ shifts observed for ultrathin polystyrene films. ${ }^{2-5}$

Figure $4 \mathrm{~b}$ shows the ratio of the characteristic length scale of the non-crosslinked filled shell to that of the corresponding bulk, $\left[\xi\left(T_{\mathrm{g}}\right)^{\mathrm{f}} /\right.$ $\left.\xi\left(T_{\mathrm{g}}\right)^{\mathrm{b}}\right]$, plotted against $d$. Here again, no distinct difference in $\xi\left(T_{\mathrm{g}}\right)$ from the bulk is observed, although a slight depression from the bulk may be discernible as a whole. The origin of this slight depression is not clear. As for the filled capsules, the polymer content is small compared with the core, resulting in a very weak $T_{\mathrm{g}}$ signal, which might lead to unknown artifacts in the data analysis. The glass transition dynamics of the non-crosslinked filled shells does not differ significantly from that in the bulk for $d \geqslant$ ca. $20 \mathrm{~nm}$.

As for the thinnest shell $(8 \mathrm{~nm}), T_{\mathrm{g}}$ is about $10 \mathrm{~K}$ lower than the bulk value, and $\xi\left(T_{\mathrm{g}}\right)$ is slightly lower (Figure 4 ). The origin of this $T_{\mathrm{g}}$ depression is not clear, but it is probably related to the same mechanism as that in ultrathin films (surface/interface and confinement effects). The radius of gyration for the PMMA material of the thinnest shell $(8 \mathrm{~nm})$ is estimated to be $10.5 \mathrm{~nm}$ in the bulk state, which exceeds the shell thickness. This might result in unstable configuration in the shell. In addition, the difference in the shell formation process may affect the resulting configuration. In the case of

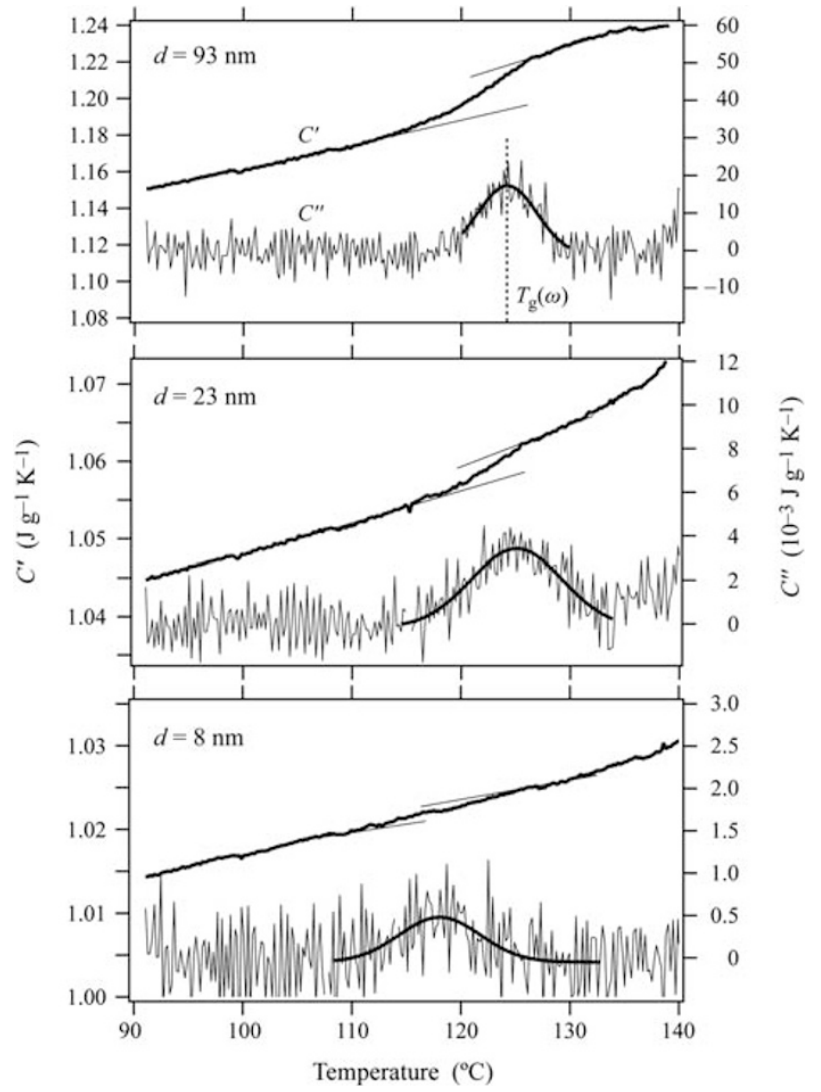

Figure 3 Typical traces for $C^{C}$ and $C^{\prime \prime}$ obtained by temperature-modulated differential scanning calorimetry cooling scans for non-crosslinked filled shells with different thicknesses. The fitted Gaussian curve for $C^{\prime \prime}(T)$ is also presented by the solid curves.

spin casting, for example, strong shear is expected to be imposed on the polymer, which may cause unstable polymer configurations. In the present deposition method, the shell is formed by simple adsorption, which is followed by evaporation of the residual monomer. Although the evaporation occurs gradually, the resulted polymer layer might be far from the equilibrium state: at the late stage of casting, the relaxation time becomes so large at a temperature well below $T_{\mathrm{g}}$ that an equilibrium state is difficult to reach. It is indisputable that the present PMMA rodlike capsules and the polystyrene shells are less sensitive to the size effects on both $T_{\mathrm{g}}$ and $\xi\left(T_{\mathrm{g}}\right)$ than ultrathin films. 

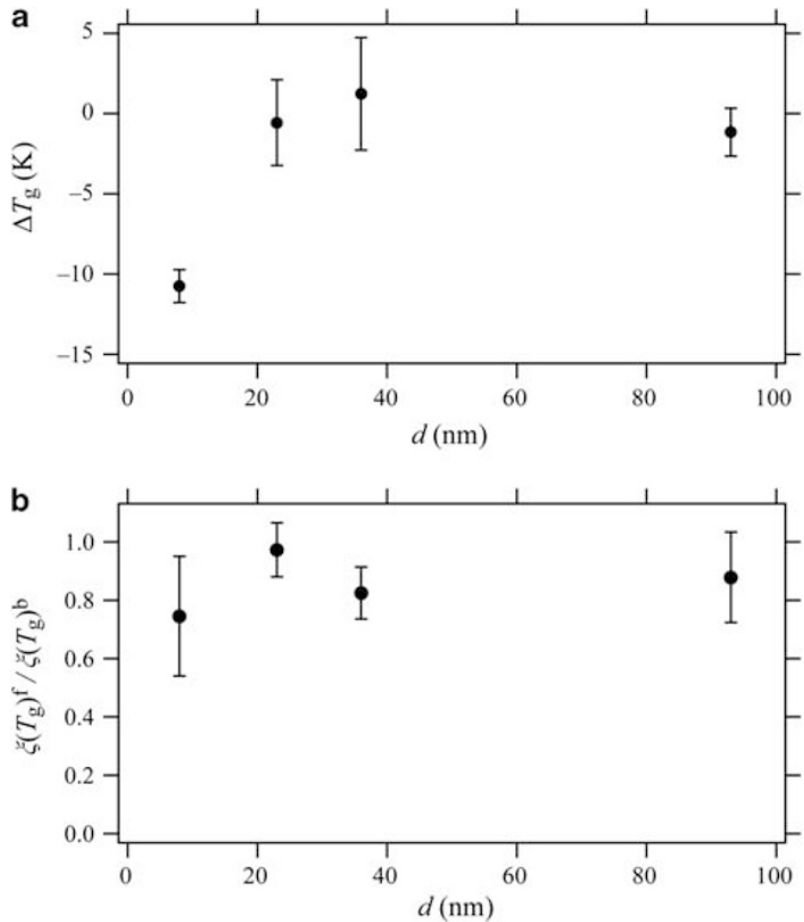

Figure 4 Deviation of $T_{\mathrm{g}}$ from the bulk $\Delta T_{\mathrm{g}}\left(=T_{\mathrm{g}}{ }^{\mathrm{f}}-T_{\mathrm{g}} \mathrm{b}\right)$ and the ratio $\xi\left(T_{\mathrm{g}}\right)^{\mathrm{f}} /$ $\xi\left(T_{\mathrm{g}}\right)^{\mathrm{b}}$ for non-crosslinked filled shells plotted against the shell thickness $d$.

Similarly, weak or no size dependence has been observed for some other systems. Polystyrene microspheres exhibit no $T_{\mathrm{g}}$ shift down to $40 \mathrm{~nm}$ in diameter, in contrast to free-standing films. ${ }^{10}$ Ultrathin films of polysulfone exhibits no size dependence of $T_{\mathrm{g}}$ down to $10 \mathrm{~nm},{ }^{27}$ and styrene-isobutylene block copolymer shows weak size dependence. ${ }^{11}$ As for the latter, the observed $T_{\mathrm{g}}$ variation has been attributed to diffuse interface.

Figure 5 shows $T_{\mathrm{g}}$ and $\xi\left(T_{\mathrm{g}}\right)$ for the shells with similar thicknesses $(17-23 \mathrm{~nm})$ with respect to the degree of crosslink $X_{\mathrm{c}} . T_{\mathrm{g}}$ increases and $\xi\left(T_{\mathrm{g}}\right)$ decreases with increasing $X_{\mathrm{c}}$. The latter seems to be a general trend. Reduction of $\xi\left(T_{\mathrm{g}}\right)$ with crosslinking corresponds to broadening of the glass transition temperature range (we confirmed this point experimentally). The observed reduction of $\xi\left(T_{\mathrm{g}}\right)$ with respect to $X_{\mathrm{c}}$ may be attributed to the heterogeneity that arises from crosslinking, and also to the reduced configurational entropy that is essential to the crosslinked structure. ${ }^{28}$ It is reasonable to assume that segments near a crosslink point have lower mobility than those sufficiently separated from crosslink points. In addition, heterogeneity in spatial distribution of the crosslink points may be responsible for the $T_{\mathrm{g}}$ broadening: as long as the difference in the reactivity of polymerization between MMA and EGDMA exists, localization of the crosslink points occurs to some extent, though we cannot characterize this feature quantitatively at present.

In Figure 5, we observe no significant difference between the filled shell and the corresponding bulk for both $T_{\mathrm{g}}$ and $\xi\left(T_{\mathrm{g}}\right)$, in contrast to the results of polystyrene rodlike shells, where $T_{\mathrm{g}}$ increases from the bulk value as the degree of crosslink increases. ${ }^{17}$ This $X_{\mathrm{c}}$ dependence for the polystyrene shells can be explained as follows: crosslinking tends to retain an unstable configuration that was established during the shell formation, while for non-crosslinked shells, the unstable configuration can readily relax to a bulk-like one during the DSC scan. Thus, we infer that in the present PMMA shells, a fairly stable
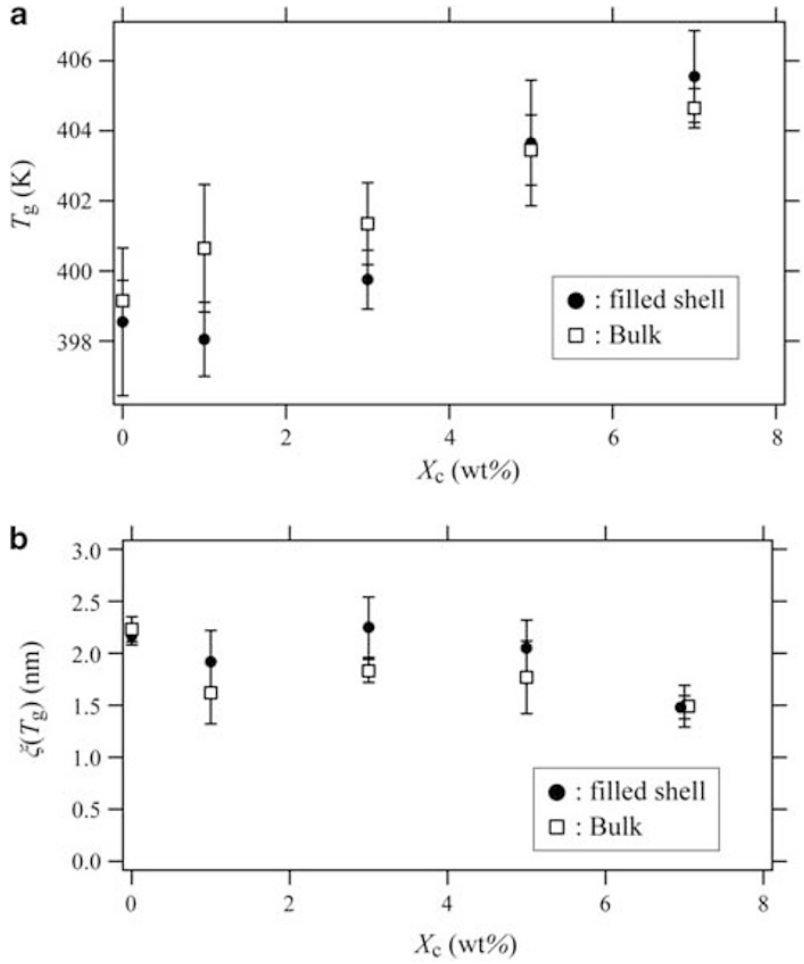

Figure $5 T_{\mathrm{g}}$ and $\xi\left(T_{\mathrm{g}}\right)$ plotted against $X_{\mathrm{c}}$ for the filled shells and bulk samples.

configuration may have already been formed during the suspension polymerization. This may be related to the polymerization mechanism: as mentioned before, MMA is more compatible (high wettability) with the $\mathrm{CaCO}_{3}$ surface than styrene, which results in a relatively stable MMA layer on the whisker surface in the suspended state. It has been argued that the $T_{\mathrm{g}}$ shift of ultrathin films depends on the interfacial tension of the polymer/substrate, ${ }^{24}$ although the density near the interface should also be taken into account to describe the interfacial effect. ${ }^{29}$ We evaluated the interfacial tension $\gamma_{\mathrm{SL}}$ for PMMA/ $\mathrm{CaCO}_{3}$ and polystyrene/ $\mathrm{CaCO}_{3}$ to be 1.5 and $2.1 \mathrm{~mJ} \mathrm{~m}^{-2}$, respectively. The $T_{\mathrm{g}}$ shift observed for crosslinked polystyrene shells may be due to the higher interfacial tension for polystyrene/ $\mathrm{CaCO}_{3}$ that leads to lower stability of the as-attached shell.

Alternatively, no $T_{\mathrm{g}}$ shift for the crosslinked PMMA shells may be attributed to an essential nature of the material that $T_{\mathrm{g}}$ is relatively insensitive to the size. For ultrathin films, in general, the thickness dependence of $T_{\mathrm{g}}$ varies prominently with the chemistry of polymer. It has been generally reported that the thickness dependence of $T_{\mathrm{g}}$ is stronger for polystyrene films than for PMMA films, ${ }^{2,26}$ and that for films of poly(vinyl acetate) with absorbed water no $T_{\mathrm{g}}$ shift is observed even for $d=23 \mathrm{~nm}$ (ref. 30). Recent theoretical studies claim that the fragility of polymers should have a negative correlation with packing efficiency, which is governed by the shape of monomer unit. ${ }^{31,32}$ PMMA has a flexible side group compared with polystyrene, which may result in higher packing efficiency. Riggleman et al. ${ }^{33}$ assume that the fragility is reduced in highly confined systems such as in ultrathin polymer films. According to this hypothesis, the confinement effect on the fragility is expected to be weaker for stronger liquids because of their low fragility even in the original bulk state. Assuming that the size dependence of the fragility is correlated to that of $T_{\mathrm{g}}$, we speculate that the size dependence of $T_{\mathrm{g}}$ is greater for polymers with higher fragility. Unfortunately, however, the reported values of Angell's 
Table 2 Glass transition temperature obtained from differential scanning calorimetry step-scan heating

\begin{tabular}{lccc}
\hline & & \multicolumn{2}{c}{$\mathrm{T}_{g}(K)$} \\
\cline { 3 - 4 } Sample & $\mathrm{X}_{c}(w t \%)$ & Filled & Hollow \\
\hline $\mathrm{B}$ & 0 & $391.8 \pm 1.0$ & $392.4 \pm 0.9$ \\
$\mathrm{E}$ & 1.0 & $398.1 \pm 1.0$ & $397.8 \pm 1.5$ \\
$\mathrm{~F}$ & 3.0 & $399.8 \pm 0.5$ & $397.9 \pm 1.0$ \\
$\mathrm{G}$ & 5.0 & $401.8 \pm 0.6$ & $401.8 \pm 0.5$ \\
$\mathrm{H}$ & 7.0 & $403.7 \pm 0.7$ & $402.7 \pm 0.5$ \\
\hline
\end{tabular}

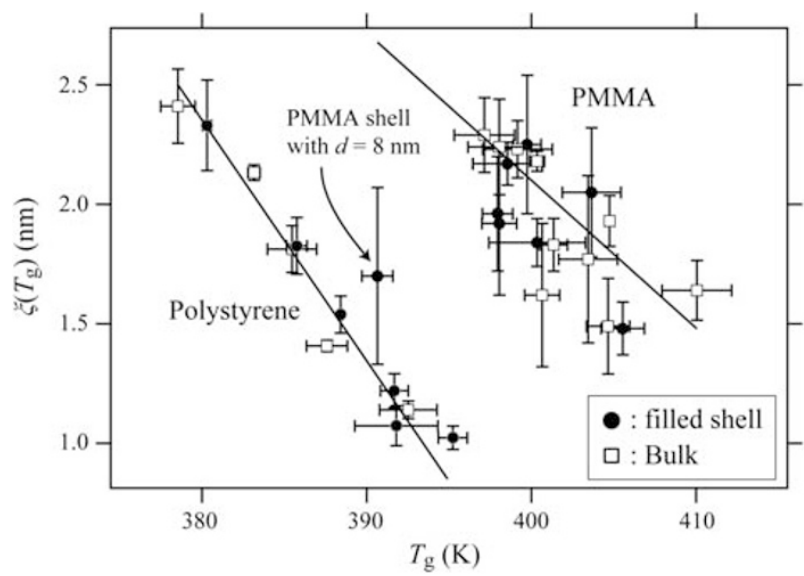

Figure $6 \xi\left(T_{\mathrm{g}}\right)$ plotted against $T_{\mathrm{g}}$ for poly(methyl methacrylate) (PMMA) and polystyrene systems. The data include filled capsules and bulk samples. The solid lines indicate the results of linear regression analysis.

dynamic fragility parameter $m$ for PMMA and polystyrene do not confirm this observation because of their widely dispersed values. ${ }^{34,35}$

Table 2 shows the glass transition temperatures obtained from the step-scan measurements for the filled and hollow shells. Little difference in $T_{\mathrm{g}}$ between the filled and hollow shells is observed. Considering the fact that $T_{\mathrm{g}}$ does not depend on $d$ for the filled shells of thicker than $20 \mathrm{~nm}$, this result may be reasonable (the hollow shell is generally thicker than the filled shell), although the status of the interface is quite different.

Figure 6 shows $\xi\left(T_{\mathrm{g}}\right)$ plotted against $T_{\mathrm{g}}$ for PMMA-filled capsules with various $X_{\mathrm{c}}$ and $d$, and for bulk samples. $\xi\left(T_{\mathrm{g}}\right)$ largely decreases with increasing $T_{\mathrm{g}}$, although considerable dispersion of the data is observed. Additionally, the shell with $d=8 \mathrm{~nm}$ does not obey the universal trend, as indicated by an arrow in Figure 6, probably due to the different mechanism of shell formation in the adsorption method as discussed before. For comparison, the data for the crosslinked polystyrene system including bulk samples are also shown in Figure 6, which exhibit a clearer decreasing feature of $\xi\left(T_{\mathrm{g}}\right)$ with respect to $T_{\mathrm{g}}$, revealing a single universal curve.

It has been demonstrated experimentally that in glass formers, dynamic heterogeneity exists without any static (structural) correlations, and the length scale of this dynamic heterogeneity increases as the temperature approaches $T_{\mathrm{g}} \cdot{ }^{36,37}$ It is pertinent to consider that the present length scale $\xi\left(T_{\mathrm{g}}\right)$ estimated by TMDSC corresponds to the length scale of dynamics heterogeneity. If we assume that $\xi\left(T_{\mathrm{g}}\right)$ corresponds to the size of CRR of the Adam-Gibbs model, ${ }^{13}$ the decrease in $\xi\left(T_{\mathrm{g}}\right)$ with increasing $T_{\mathrm{g}}$ shown in Figure 6 implies that the segmental relaxation time $\tau$ is not a unique function of the size of
CRR. In other words, the same absolute value of $\tau$, which is typically $10^{2}-10^{3} \mathrm{~s}$ at $T_{\mathrm{g}}$, can be associated with different sizes of the CRR depending upon the degree of crosslink, shell thickness and status of interface.

As for the PMMA system, $T_{\mathrm{g}}$ variation is actually due to the variation of $X_{\mathrm{c}}$ because $T_{\mathrm{g}}$ is almost independent of the shell thickness and of the status of interface, except for the thinnest $(8 \mathrm{~nm})$ shell. Thus, the trend in Figure 6 implies that the higher the density of crosslink, the smaller the CRR at $T_{\mathrm{g}}$. This may be explained as follows: when crosslinking occurs, the CRR at $T_{\mathrm{g}}$ has to become smaller to keep the relaxation time as $\tau\left(T_{\mathrm{g}}\right)=10^{2}-10^{3} \mathrm{~s}$. The slope of the profile, which actually represents the $X_{\mathrm{c}}$ dependence of $\xi\left(T_{\mathrm{g}}\right)$ in the case of the PMMA system, is considered to be the manifestation of the crosslink effect on the relaxation time. Linear regression analysis shown by the solid lines in Figure 6 reveals that the slope is greater for the polystyrene system than for the PMMA system. As for the polystyrene system, $\xi\left(T_{\mathrm{g}}\right)$ depends not only on $X_{\mathrm{c}}$ but also on the shell thickness. However, the present results might show that the crosslink effect on the segmental relaxation time is rather strong for the polystyrene system. This may be due to the stiff feature of the polystyrene chain in the amorphous state.

The results in Figure 6 also seem to be compatible with both the Adam-Gibbs and the random first-order transition theories. ${ }^{13,15}$ According to the Adam-Gibbs theory, $\xi\left(T_{\mathrm{g}}\right)$ can be written as

$$
\xi\left(T_{\mathrm{g}}\right)^{3}=\ln \left[\frac{\tau\left(T_{\mathrm{g}}\right)}{\tau_{0}}\right] \frac{k T_{\mathrm{g}}}{\Delta \mu},
$$

where $\Delta \mu$ is the activation energy for cooperative rearrangement per unit volume, $\tau_{0}$ is the prefactor which determines the absolute value of the relaxation time, and $k$ is the Boltzmann constant. Given that the profile in Figure 6 is due to the crosslink effect, one can draw a quite reasonable conclusion that both $\tau_{0}$ and $\Delta \mu$ increase with $X_{\mathrm{c}}$ under the condition that $\tau\left(T_{\mathrm{g}}\right)$ is constant. According to the recent argument based on the random first-order transition theory, ${ }^{37}$ on the other hand, $\ln \left(\tau / \tau_{0}\right)$ is expressed by a universal increasing function $F$ of the configurational entropy of a CRR as

$$
\ln \left[\frac{\tau\left(T_{\mathrm{g}}\right)}{\tau_{0}}\right]=F\left[\frac{\xi\left(T_{\mathrm{g}}\right)^{3} S_{\mathrm{c}}\left(T_{\mathrm{g}}\right)}{k}\right],
$$

where $S_{\mathrm{c}}$ is the configurational entropy per unit volume, and thus corresponds to the configurational entropy of a CRR. Given that both $\xi^{3}$ and $S_{\mathrm{c}}$ decrease with increasing $X_{\mathrm{c}}$, the prefactor $\tau_{0}$ should increase with $X_{\mathrm{c}}$.

\section{CONCLUSIONS}

In this study, we successfully synthesized various rodlike capsules consisting of a calcium carbonate core and a PMMA-based polymer shell by two methods: suspension polymerization, and the simple adsorption method. We investigated glass transition dynamics of the obtained thin PMMA shells by use of the TMDSC technique and revealed the following points:

1. For non-crosslinked shells of $d \geqslant 23 \mathrm{~nm}, T_{\mathrm{g}}$ and $\xi\left(T_{\mathrm{g}}\right)$ are approximately the same as those of the bulk samples and are independent of the shell thickness.

2. A non-crosslinked shell with $d=8 \mathrm{~nm}$ shows a reduced $T_{\mathrm{g}}$ from the bulk value. The result may be attributed to the different mechanism of shell formation and to the very small value of $d$.

3. Crosslinked PMMA shells do not show $T_{\mathrm{g}}$ deviation from the bulk up to $X_{\mathrm{c}}=7.0 \%$, in contrast to the results for the polystyrene shells. The result may be attributed to different stability of the 
shell as revealed by the difference in interfacial energy and/or to the different efficiency of segment packing that arises from the chemical structure.

4. Hollow shells exhibit neither $T_{\mathrm{g}}$ nor $\xi\left(T_{\mathrm{g}}\right)$ shifts from the bulk for $d \geqslant$ ca. $20 \mathrm{~nm}$.

5. $\xi\left(T_{\mathrm{g}}\right)$ exhibits a universal profile that decreases with increasing $T_{\mathrm{g}}$, which may be interpreted as the crosslink effect on the size of CRR at $T_{\mathrm{g}}$.

\section{ACKNOWLEDGEMENTS}

This work was supported by a Grant-in-Aid for Scientific Research (no. 18550107) from the Ministry of Education, Culture, Sports, Science and Technology of Japan. We thank Toshiya Nakamoto for help in sample preparations, and also thank Maruo Calcium, Japan, for supplying the calcium carbonate materials.

1 Alcoutlabi, M. \& McKenna, B. Effects of confinement on material behaviour at the nanometre size scale. J. Phys. Condens. Matter 17, R461-R524 (2005).

2 Forrest, J. A. \& Dalnoki-Veress, K. The glass transition in thin polymer films. Adv. Colloid Interface Sci. 94, 167-196 (2001).

3 Fukao, K. \& Miyamoto, Y. Slow dynamics near glass transitions in thin polymer films. Phys. Rev. E 64, 011803 (2001).

4 Efremov, M.Yu., Olson, E. A., Zhang, M., Zhang, Z. \& Allen, L. H. Probing glass transition of ultrathin polymer films at a time scale of seconds using fast differential scanning calorimetry. Macromolecules 37, 4607-4616 (2004).

5 Efremov, M.Yu., Kiyanova, A. V. \& Nealey, P. F. Temperature-modulated ellipsometry: a new probe for glass transition in thin supported polymer films. Macromolecules 41, 5978-5980 (2008).

6 Serghei, A., Huth, H., Schick, C. \& Kremer, F. Glassy dynamics in thin polymer layers faving a free upper interface. Macromolecules 41, 3636-3639 (2008).

7 Tress, M., Erber, M., Mapesa, E. U., Huth, H., Jan Müller, J., Serghei, A., Schick, C. Eichhorn, K.- J., Voit, B. \& Kremer, F. Glassy dynamics and glass transition in nanometric thin layers of polystyrene. Macromolecules 43, 9937-9944 (2010).

8 Arndt, M., Stannarius, R., Groothues, H., Hempel, E. \& Kremer, F. Length scale of cooperativity in the dynamic glass transition. Phys. Rev. Lett. 79, 2077-2080 (1997).

9 Park, J.- Y. \& McKenna, G. B. Size and confinement effects on the glass transition behavior of polystyrene/o-terphenyl polymer solutions. Phys. Rev. B 61, 6667-6676 (2000).

10 Sasaki, T., Shimizu, A., Mourey, T. H., Thurau, C. T. \& Ediger, M. D. Glass transition of small polystyrene spheres in aqueous suspensions. J. Chem. Phys. 119, 8730-8735 (2003).

11 Robertson, C. G., Hogan, T. E., Rackaitis, M., Puskas, J. E. \& Wang, X. Effect of nanoscale confinement on glass transition of polystyrene domains from self-assembly of block copolymers. J. Chem. Phys. 132, 104904 (2010).

12 Sasaki, T., Kitagawa, T., Sato, S., Irie, S. \& Sakurai, K. Core/shell and hollow polymeric capsules prepared from calcium carbonate whisker. Polym. J. 37, 434-438 (2005).

13 Adam, G. \& Gibbs, J. H. On the temperature dependence of cooperative relaxation properties in glass-forming liquids. J. Chem. Phys. 43, 139-146 (1965).

14 Hutchinson, J. M., Montserrat, S., Calventus, Y. \& Cortes, P. Application of the AdamGibbs equation to the non-equilibrium glassy state. Macromolecules 33, 5252-5262 (2000).
15 Lubchenko, V. \& Wolynes, P. G. Theory of structural glasses and supercooled liquids. Ann. Rev. Phys. Chem. 58, 235-266 (2006).

16 Sasaki, T, Kawagoe, S. Mitsuya, H. Irie, S. \& Sakurai, K. Glass transition of crosslinked polystyrene shells formed on the surface of calcium carbonate whisker. J. Polym. Sci. B Polym. Phys. 44, 2475-2485 (2006).

17 Sasaki, T., Misu, M., Shimada, T. \& Teramoto, M. Glass transition and its characteristic length for thin crosslinked polystyrene shells of rodlike capsules. J. Polym. Sci. B Polym. Phys. 46, 2116-2125 (2008).

18 Matyjaszewski, K. \& Xia, J. Atom transfer radical polymerization. Chem. Rev. 101, 2921-2990 (2001).

19 Ohno, K., Morinaga, T., Koh, K., Tsujii, Y. \& Fukuda, T. Synthesis of monodisperse silica particles coated with well-defined, high-density polymer brushes by surface-initiated atom transfer radical polymerization. Macromolecules 38, 2137-2142 (2005).

20 Hemmpel, E., Hensel, A., Schick, C. \& Donth, E. Characteristic length of dynamic glass transition near Tg for a wide assortment of glass-forming substances. J. Phys. Chem. B 104, 2460-2466 (2000).

21 Pyda, M. Ed. ATHAS Data Bank; http://athas.prz.rzeszow.pl.

22 van Oss, C. J., Chaudhury, M. K. \& Good, R. Monopolar surfaces. Adv. Colloid Interface Sci. 28, 35-64 (1987)

23 Lee, L.- H. Correlation between Lewis acid-base surface interaction components and linear solvation energy relationship solvatochromic $\alpha$ and $\beta$ parameters. Langmuir $\mathbf{1 2}$ 1681-1687 (1996).

24 Fryer, D. S., Peters, R. D., Kim, E. J., Tomaszewski, J. E., de Pablo, J. J., Nealey, P. F., White, C. C. \& Wu, W. Dependence of the glass transition temperature of polymer films on interfacial energy and thickness. Macromolecules 34, 5627-5634 (2001).

25 Peters, R. D., Yang, X. M., Kim, T. K., Sohn, B. H. \& Nealey, P. F. Using self-assembled monolayers exposed to $\mathrm{X}$-rays to control the wetting behavior of thin films of diblock copolymers. Langmuir 16, 4625-4631 (2000).

26 Fryer, D. S., Nealey, P. F. \& de Pablo, J. J. Thermal probe measurements of the glass transition temperature for ultrathin polymer films as a function of thickness. Macromolecules 33, 6439-6447 (2000).

27 Labahn, D., Mix, R. \& Schönhals, A. Dielectric relaxation of ultrathin films of supported polysulfone. Phys. Rev. E 79, 011801 (2009).

28 Sasaki, T., Uchida, T. \& Sakurai, K. Effect of crosslink on the characteristic length of glass transition of network polymers. J. Polym. Sci. B Polym. Phys. 44, 1958-1966 (2006).

29 Tsui, O. K. C., Russell, T. P. \& Hawker, C. J. Effect of interfacial interactions on the glass transition of polymer thin films. Macromolecules 34, 5535-5539 (2001).

30 Kim, S., Mundra, M. K., Roth, C. B. \& Torkelson, J. M. Suppression of the Tg-nanoconfinement effect in thin poly(vinyl acetate) films by sorbed water. Macromolecules 43, 5158-5161 (2010).

31 Dudowicz, J., Freed, K. F. \& Douglas, J. F. The glass transition temperature of polymer melts. J. Phys. Chem. B 109, 21285-21292 (2005).

32 Dudowicz, J., Freed, K. F. \& Douglas, J. F. Fragility of glass-forming polymer liquids. J. Phys. Chem. B 109, 21350-21356 (2005).

33 Riggleman, R. A., Yoshimoto, K., Douglas, J. F. \& de Pablo, J. J. Influence of confinement on the fragility of antiplasticized and pure polymer films. Phys. Rev. Lett 97, 045502 (2006).

34 Böhmer, R., Ngai, K. L., Angell, C. A. \& Plazek, D. J. Nonexponential relaxations in strong and fragile glass formers. J. Chem. Phys. 99, 4201-4209 (1993).

35 Huang, D. \& McKenna, G. B. New insights into the fragility dilemma in liquids. J. Chem. Phys. 114, 5621-5630 (2001); and references therein.

36 Berthier, L., Biroli, G., Bouchaud, J.- P., Cipelletti, L., Masri, D.El., L'Hôte, D., Ladieu, F. \& Pierno, M. Direct experimental evidence of a growing length scale accompanying the glass transition. Science 310, 1797-1800 (2005).

37 Capaccioli, S., Ruocco, G. \& Zamponi, F. Dynamically correlated regions and configurational entropy in supercooled liquids. J. Phys. Chem. B 112, 10652-10658 (2008). 